\title{
Investigation of the
}

\section{Autoregulator-Receptor System in the Pristinamycin Producer Streptomyces pristinaespiralis}

\author{
Franziska Handel ${ }^{1}$, Andreas Kulik ${ }^{1}$ and Yvonne Mast ${ }^{1,2,3,4 *}$
}

${ }^{1}$ Department of Microbiology/Biotechnology, Faculty of Science, Interfaculty Institute of Microbiology and Infection Medicine, University of Tübingen, Tübingen, Germany, ${ }^{2}$ German Center for Infection Research (DZIF), Partner Site Tübingen, Tübingen, Germany, ${ }^{3}$ Department Bioresources for Bioeconomy and Health Research, Leibniz Institute DSMZ - German Collection of Microorganisms and Cell Cultures, Braunschweig, Germany, ${ }^{4}$ Department of Microbiology, Technical University Braunschweig, Braunschweig, Germany

\section{OPEN ACCESS}

Edited by:

Christian Sohlenkamp,

National Autonomous University of Mexico, Mexico

Reviewed by:

Yinhua Lu,

Shanghai Normal University, China

Paloma Liras,

Universidad de León, Spain

*Correspondence:

Yvonne Mast

yvonne.mast@dsmz.de

Specialty section:

This article was submitted to Microbial Physiology and Metabolism, a section of the journal

Frontiers in Microbiology

Received: 14 July 2020 Accepted: 10 September 2020 Published: 30 September 2020

Citation:

Handel F, Kulik A and Mast Y

(2020) Investigation of the Autoregulator-Receptor System in the Pristinamycin Producer

Streptomyces pristinaespiralis.

Front. Microbiol. 11:580990. doi: 10.3389/fmicb.2020.580990
Pristinamycin biosynthesis in Streptomyces pristinaespiralis is governed by a complex hierarchical signaling cascade involving seven different transcriptional regulators (SpbR, PapR1, PapR2, PapR3, PapR4, PapR5, and PapR6). The signaling cascade is triggered by $\gamma$-butyrolactone (GBL)-like effector molecules, whereby the chemical structure of the effector, as well as its biosynthetic origin is unknown so far. Three of the pristinamycin transcriptional regulators (SpbR, PapR3, and PapR5) belong to the type of $\gamma$-butyrolactone receptor (GBLR). GBLRs are known to either act as "real" GBLRs, which bind GBLs as ligands or as "pseudo" GBLRs binding antibiotics or intermediates thereof as effector molecules. In this study, we performed electromobility shift assays (EMSAs) with SpbR, PapR3, and PapR5, respectively, in the presence of potential ligand samples. Thereby we could show that all three GBLRs bind synthetic 1,4-butyrolactone but not pristinamycin as ligand, suggesting that SpbR, PapR3, and PapR5 act as "real" GBLRs in S. pristinaespiralis. Furthermore, we identified a cytochrome P450 monooxygenase encoding gene snbU as potential biosynthesis gene for the GBLR-interacting ligand. Inactivation of snbU resulted in an increased pristinamycin production, which indicated that SnbU has a regulatory influence on pristinamycin production. EMSAs with culture extract samples from the snbU mutant did not influence the target binding ability of SpbR, PapR3, and PapR5 anymore, in contrast to culture supernatant samples from the S. pristinaespiralis wild-type or the pristinamycin deficient mutant papR2::apra, which demonstrates that SnbU is involved in the synthesis of the GBLR-interacting ligand.

Keywords: Streptomyces, antibiotics, transcriptional regulation, signal molecules, $\gamma$-butyrolactone

\footnotetext{
Abbreviations: ACT, actinorhodin; A-factor, ligand from S. griseus; $\mathrm{Apr}^{\mathrm{R}}$, apramycin resistance cassette; ARE, autoregulatory element; BL, 1,4-butyrolactone; EMSA, electromobility shift assays; HPLC, high performance liquid chromatography; HTH, helix-turn-helix; GBL, $\gamma$-butyrolactone; GBLR, $\gamma$-butyrolactone receptor; pGBLR, pseudo $\gamma$-butyrolactone receptor; GDL, $\gamma$-decanolactone; LB, Luria-Bertani; $I$, isoelectric point; PIA, pristinamycin IA; PIIA, pristinamycin IIA; RED, undecylprodigiosin; RT-PCR, Reverse transcriptase-polymerase chain reaction; SARP, Streptomyces antibiotic regulatory protein; SCB, ligands from S. coelicolor; SVB, ligands from S. venezuelae; SpbR, S. pristinaespiralis butyrolactone-responsive transcriptional repressor; TetR, Tetracycline repressor protein; VB, virginiae butanolide; ligand from S. virginiae; WT, wild-type; YEME, yeast extract, malt extract.
} 


\section{INTRODUCTION}

Bacteria of the genus Streptomyces are filamentous, grampositive soil bacteria, which are characterized by a complex morphological life-cycle and their ability to produce a wide variety of secondary metabolites, including the majority of all known antibiotics (Tanaka and Omura, 1990; Liu et al., 2013). Antibiotic production in these organisms is usually subject to control through complex regulatory networks, which respond to various environmental and physiological factors (McCormick and Flärdh, 2012). One common important initiation principle that triggers antibiotic production in streptomycetes is governed by a quorum sensing-like system employing $\gamma$-butyrolactones (GBLs) as signaling molecules. GBLs are small, diffusible molecules referred to as microbial hormones, which are produced by at least $60 \%$ of all Streptomyces species, where they induce antibiotic production in nanomolar concentrations (Takano et al., 2000; Liu et al., 2013; Niu et al., 2016). Depending on the type of GBL, the molecules are synthesized by the action of only one, or multiple enzymes: e.g., AfsA synthesizes the A-factor GBL in the streptomycin producer Streptomyces griseus (Ando et al., 1997) and ScbA is responsible for the synthesis of the SCBs in the actinorhodin (ACT)/undecylprodigiosin (RED) producer Streptomyces coelicolor (Hsiao et al., 2007), whereby three enzymes (BarX, BarS1, and BarS2) have been shown to be involved in virginiae butanolide (VB) biosynthesis in the virginiamycin producer Streptomyces virginiae (Shikura et al., 2002; Lee et al., 2008, 2010). On the molecular level, GBLs bind to their cognate GBL receptor (GBLR), which resemble TetRlike family regulators that usually act as transcriptional repressors by binding to specific sequence motifs (ARE motifs) within the promoter regions of their target genes. TetR-like regulators bind to the DNA regions as " $\Omega$ " shaped dimers (Ramos et al., 2005). They consist of a helix-turn-helix (HTH) DNA-binding motif in the $\mathrm{N}$-terminal domain, and a ligand-binding pocket in the C-terminal domain (Yu et al., 2010). Upon GBL binding, the GBLR undergoes a conformational change, whereby it is released from the promoter region(s), which in turn allows transcription of the previously repressed gene(s) (Ramos et al., 2005). Wellknown examples for GBLR regulators in streptomycetes are ArpA from S. griseus, which binds the A-factor as a ligand or ScbR from S. coelicolor responding to the SCB1 molecule (Takano et al., 2008). GBLR target genes often involve additional transcriptional regulatory genes, which ends up in quite complex hierarchical signaling cascades employing pleiotropic but also pathway-specific transcriptional regulators (Bibb, 2005).

Besides the above described "real" GBLR regulators also homologous regulator-types are present in streptomycetes called "pseudo" GBLRs (pGBLRs), which instead of GBLs bind antibiotics or antibiotic intermediates as ligands ( $\mathrm{Xu}$ et al., 2010). The first described and best characterized pGBLRs are ScbR2 from S. coelicolor and JadR2 from Streptomyces venezuelae, which both bind endogenous antibiotics from these bacteria as ligands, as there are: ACT and RED in terms of ScbR2, and jadomycin and chloramphenicol in terms of JadR2 (Xu et al., 2010; Table 1). Interestingly, these antibiotics bind to their associated receptors even if they have a complete distinct chemical structure. The pGBLRs act in concert with the genuine GBLRs in these strains, which are ScbR in S. coelicolor, with the associated SCB ligands (Xu et al., 2010) and JadR1/3 in S. venezuelae, with the cognate SVB ligands (Zou et al., 2014). Together they control the transcriptional regulation of the respective antibiotic biosynthesis. Further examples of such GBLR/pGBLR combinations have been suggested for the virginiamycin producer S. virginiae (Table 1). Here, BarA has been shown to function as the GBLR, which binds VBs as ligands, whereas BarB is the proposed pGBLR (Matsuno et al., 2004). Accordingly, SagR is the suggested GBLR in the auricin producer Streptomyces aureofaciens and Aur1R the pGBLR (Mingyar et al., 2015). In Streptomyces lavendulae, which produces the blue pigment indigoidine, cycloserine and nucleoside antibiotics, FarA is the GBLR that binds the IM-2 effector molecule, whereas FarR2 is the suggested pGBLR (Kurniawan et al., 2016). Based on phylogenetic comparisons between different GBLRlike regulators from streptomycetes, BulR1 from the tacrolimus (FK506) producer Streptomyces tsukubaensis is the proposed GBLR and BulR2 the pGBLR (Salehi-Najafabadi et al., 2014). This assignment has also been done due to the different $\mathrm{p} I$ values of both regulator types (BulR1: $\mathrm{p} I$ 5.51; BulR2: $\mathrm{p} I$ 10.15). $\mathrm{p} I$ differences have previously been suggested to serve as a criterion to classify GBLRs and pGBLRs, whereby GBLRs tend to have a more acidic $\mathrm{p} I$ value and pGBLRs a more basic one (Lee et al., 2008; Kitani et al., 2008). This was also observed for the GBLR/pGBLR combination BarA/BarB of $S$. virginiae (Kinoshita et al., 1997; Kawachi et al., 2000) or FarA/FarR2 of S. lavendulae (Kawachi et al., 2000; Kitani et al., 2008). In the various antibiotic producers GBLRs and pGBLRs act in concert and together fine-tune secondary metabolite production, which can end up in complex feed-forward/feedback loops (Xu and Yang, 2019).

Such a complex regulatory system is also present in the pristinamycin producer Streptomyces pristinaespiralis (Mast et al., 2011, 2015). Pristinamycin is a streptogramin antibiotic consisting of the peptide antibiotic pristinamycin I and the polyketide antibiotic pristinamycin II (Mast and Wohlleben, 2014). Regulation of pristinamycin biosynthesis is governed by a GBL quorum sensing-like system (Mast et al., 2015). So far, the GBLR-interacting ligand molecule(s) as well as its encoding biosynthetic gene(s) have not been identified. In a previous study, endogenous factors have been isolated from S. pristinaespiralis cultures, which showed an inducing effect on pristinamycin production (Paquet et al., 1992). Furthermore, the addition of the A-factor, as well as several commercial GBLs resulted in an induction of pristinamycin production in S. pristinaespiralis, whereby the A-factor showed the strongest inducing effect (Paquet et al., 1992). Thus, it has been suggested that the pristinamycin-specific effector may resemble a A-factorlike GBL (Paquet et al., 1992). The reported cognate GBLR in $S$. pristinaespiralis is SpbR (S. pristinaespiralis butyrolactoneresponsive transcriptional repressor) (Folcher et al., 2001), which acts as the pleiotropic regulator of the pristinamycin signaling cascade (Mast et al., 2015).

The signaling cascade comprises six additional pristinamycinspecific transcriptional regulators, which are encoded by 
TABLE 1 | GBLRs and pGBLRs from different antibiotic-producing streptomycetes.

\begin{tabular}{|c|c|c|c|c|}
\hline Strain & GBLR & pGBLR & Antibiotic & References \\
\hline S. acidiscabies & SabR & SabS & Thaxtomin & Kitani et al., 2008 \\
\hline S. ambofaciens ${ }^{*}$ & AlpZ & AlpW & Alpomycin & Bunet et al., 2011 \\
\hline S. ansochromogenes & SabR1 & SabR2 & Nikkomycin & Wang et al., 2018 \\
\hline S. aureofaciens* & SagR & Aur1R & Auricin & Wang et al., 2011 \\
\hline S. avermitilis* & AvaR1, AvaR3 & AvaR2 & Avermectin & Zhu et al., 2016 \\
\hline S. coelicolor ${ }^{*}$ & ScbR & ScbR2, CprB & Actinorhodin, Undecylprodigiosin & Xu et al., 2010 \\
\hline S. fradiae & TylP & TylQ & Tylosin & Bignell et al., 2007 \\
\hline S. griseus & ArpA & SGR6382 & Streptomycin & Horinouchi, 2007 \\
\hline S. lavendulae* & FarA & FarR2 & Indigoidine, Cycloserine, Nucleoside antibiotics & Kurniawan et al., 2016 \\
\hline S. rochei & SrrA & SrrB & Lankamycin & Arakawa et al., 2012 \\
\hline S. scabies* & SscR & SscF & Thaxtomin & Kitani et al., 2008 \\
\hline S. tsukubaensis* & BulR1 & BulR2 & Tacrolimus & Salehi-Najafabadi et al., 2014 \\
\hline S. venezuelae* & JadR1/3 & JadR2 & Jadomycin & Zou et al., 2014 \\
\hline S. virginiae & BarA & BarB & Virginiamycin & Nishida et al., 2007 \\
\hline
\end{tabular}

Experimentally verified $p G B L R$ function is marked with an asterisk.

the genes $p a p R 1, p a p R 2, p a p R 3, p a p R 4, p a p R 5$, and $p a p R 6$ (Mast et al., 2015; Figure 1). papR1, 2, 4, and 6 code for transcriptional activators, whereby the encoded PapR1, 2, and 4 proteins belong to the SARP-type family of transcriptional regulators and PapR6 represents a response regulator. papR3 and papR5 encode the TetR-like regulators PapR3 and PapR5, which act as transcriptional repressors of pristinamycin biosynthesis (Mast et al., 2015). The regulatory function of the pristinamycin regulators has been shown by mutagenesis and overexpression experiments, as well as RT-PCR and EMSA studies (Mast et al., 2015). Regarding the pristinamycin-specific TetR-like regulators, inactivation of $p a p R 3$ and papR5

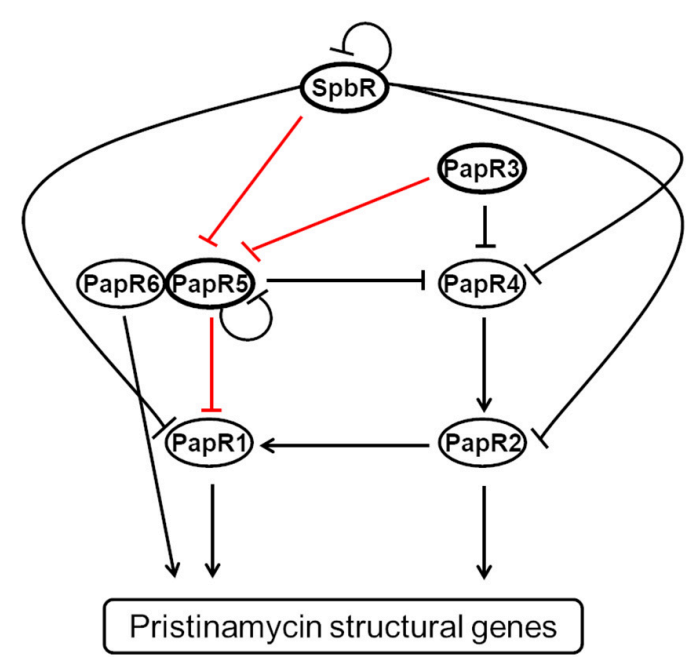

FIGURE 1 | Model of the regulation of the pristinamycin regulatory signaling cascade of S. pristinaespiralis according to Mast et al. (2015). Binding of pristinamycin regulators is indicated by arrows (activating effect) and perpendicular lines (repressive effect). The GBLR-like regulators SpbR, PapR3, and PapR5 used in this study are highlighted by bold lined ellipses. The tested promoter interactions are indicated as red lines. resulted in a up to 150 and $300 \%$ increase of pristinamycin production, respectively (Mast et al., 2015). The papR5 mutant additionally showed a morphological defect and lacked the formation of aerial mycelium and spores, which illustrated the pleiotropic regulatory function of the encoded PapR5 regulator (Mast et al., 2015).

On the molecular level, SpbR binds to its own promoter region, as well as to the promoter regions of nearly all $p a p R$ regulators, except for $p a p R 3$ and $p a p R 6$. PapR3 binds to the promoter regions of $p a p R 5$ and $p a p R 4$, whereas PapR5 binds to its own promoter region and to the promoter region of the SARP gene $p a p R 1$. papR1 transcription is also under control of the SARP-type regulator PapR2, which is the essential activator of pristinamycin biosynthesis that binds to promoter regions of the pristinamycin I and II structural genes (Mast et al., 2015; Figure 1). It has been found that all GBLR-like regulators SpbR, PapR3, and PapR5 have a conserved Trp residue at position 191, which usually serves as a binding site for GBLs in homologous known GBL receptors (Mast, 2008), suggesting that all three regulators may act as GBLRs. According to the classification of Salehi-Najafabadi et al. (2014) PapR3 with a basic pI of 9.92 would be a pGBLR, whereas SpbR and PapR5 with an acidic $\mathrm{p} I$ of 5.72 and 6.08, respectively, should be "real" GBLRs (Mast et al., 2015). However, this association is in conflict with a phylogenetic study of various TetR-like regulators from different streptomycetes, according to which PapR5 was assigned to the pGBLRs and SpbR and PapR3 to GBLRs (Cuthbertson and Nodwell, 2013). Thus, experimental research is necessary to ascertain if SpbR, PapR3, and PapR5 act as "real" or "pseudo" GBLRs in $S$. pristinaespiralis.

Here, we report on EMSA studies with SpbR, PapR3, and PapR5 in the presence of various potential ligand samples, such as commercial GBLs, pristinamycin or S. pristinaespiralis culture extract samples and thereby demonstrate that all three GBLR-like regulators act as real GBLRs in S. pristinaespiralis. Furthermore, we report on the identification and functional analysis of the potential GBLR-interacting ligand biosynthesis gene $s n b U$. 


\section{RESULTS}

\section{SpbR, PapR3, and PapR5 Are GBLR-Like Regulators With DNA Binding Ability}

In a previous study it has been shown that SpbR, PapR3, and PapR5 from S. pristinaespiralis are TetR-like regulators that bind to promoter regions of pristinamycin regulatory genes (Mast et al., 2015; Figure 1). In order to perform liganddependent regulator-DNA interaction analysis, the experimental conditions had to be predefined in order to allow for proper regulator-DNA interaction studies. For these analyses EMSAs were performed with the GBLR-type regulators SpbR, PapR3, and PapR5 together with representative cognate promoter regions. PpapR5 was used as a representative promoter region for SpbR and PapR3, whereas PpapR1 served as test promoter fragment for PapR5 (Supplementary Figure S1). For EMSA studies, cell lysates from the respective Streptomyces lividans overexpression strains SLspbR-OE, SLpapR3-OE, and SLpapR5$O E$ expressing the GBLRs as His-tagged proteins were used together with the 276 and 291 bp Cy5-labeled promoter regions PpapR1 and PpapR5, respectively, (Supplementary Table S1 and Supplementary Figure S1, band 3). The respective promoter fragment without the addition of lysate served as internal reference (Supplementary Figure S1, band 1). As a control, the shifts were carried out with cell lysates from S. lividans harboring the empty pGM190 vector (SLPGM190) (Supplementary Table S1 and Supplementary Figure S1, band 2). Shift specificity was verified by the addition of increasing concentrations of competitive unlabeled target DNA, which successively out-competed the specific binding interaction (Supplementary Figure S1, band 4-6), as well as competitive unspecific DNA, which did not influence binding interaction (Supplementary Figure S1, band 7). EMSA assays revealed that SpbR and PapR3 cell lysate samples lead to a specific shift of the PpapR5 promoter fragment, whereas the PapR5 cell lysate samples led a shifted PpapR1 band (Supplementary Figure S1, band 3). Thus, EMSAs verified that SpbR and PapR3 specifically bound to the promoter region of papR5, and PapR5 specifically bound to the promoter region of papR1.

\section{DNA Binding Activity of Pristinamycin-Specific GBLR-Like Regulators Is Affected by Synthetic 1,4-Butyrolactone}

In a previous study from Paquet et al. (1992) it has been shown that pristinamycin biosynthesis is induced by the addition of different commercial GBLs. In order to ascertain if the pristinamycin-specific GBLR-like regulators function as receptors for GBLs as ligands, EMSAs were performed with SpbR, PapR3, and PapR5 in the presence of selected commercially available GBLs. As representative GBLs, synthetic 1,4-butyrolactone (BL) $\left(\mathrm{C}_{4} \mathrm{H}_{6} \mathrm{O}_{2}\right.$, Figure 2A) and $\gamma$-decanolactone (GDL) $\left(\mathrm{C}_{10} \mathrm{H}_{18} \mathrm{O}_{2}\right.$, Figure 2B) were tested. $\mathrm{BL}$ was used as a synthetic A-factor analog. A-factor and GDL both induced pristinamycin production in S. pristinaespiralis at minimal concentrations ( 0.001 and $0.25 \mathrm{~g} / \mathrm{ml}$, respectively) before (Paquet et al., 1992). Furthermore, BL has been shown to act as inductor of validamycin production in Streptomyces hygroscopicus 5008 (Tan et al., 2013) and bitespiramycin production in Streptomyces spiramyceticus (Gao et al., 2019). EMSAs were carried out with lysates of SLspbR-OE and SLpapR3$O E$ together with PpapR5 and SLpapR5-OE with PpapR1 as reported above. In addition to the EMSA samples containing the GBLR lysate and the respective test promoter region, increasing concentrations $(1,2$, and $4 \mu \mathrm{l})$ of BL and GDL were added, respectively. EMSA analysis revealed that the addition of $1 \mu \mathrm{l} \mathrm{BL}$ to the GBLR test samples eliminated the presence of the shifted bands in samples with SpbR, PapR3, and PapR5, respectively, whereas addition of up to $4 \mu \mathrm{l}$ GDL did not (Figure 2C). This suggests that BL, but not GDL, acts as artificial ligand for the pristinamycin-specific GBLRs. Since addition of BL prevented the GBLR-DNA binding ability of SpbR, PapR3, and PapR5, this may indicate that the pristinamycin-specific GBLR-like regulators act as "real" GBLRs. GBLs are reported to be highly specific for their cognate GBLRs. The lactone ring is a structural characteristic and essential for the functionality of the GBLs. The synthetic GBLs BL and GDL used here only differ in the additional C6-fatty acid side chain present in GDL (Figure 2A vs. Figure 2B). This suggests that the in vitro binding of GDL to the receptors is prevented due to the long fatty acid side chain.

\section{Addition of BL Induces Pristinamycin Production in S. pristinaespiralis}

As found from the above mentioned EMSA studies, BL serves as an artificial ligand for the pristinamycin-specific GBLR-like regulators (Figure 2). In a previous study it has been shown that external addition of synthetic GBLs to the S. pristinaespiralis culture induces pristinamycin biosynthesis (Paquet et al., 1992). Furthermore, addition of BL induced validamycin and bitespiramycin production before (Tan et al., 2013; Gao et al., 2019). Thus, it can be expected that addition of the artificial ligand $\mathrm{BL}$ to the $S$. pristinaespiralis culture induces pristinamycin production. To investigate whether addition of synthetic BL has an inducing effect on pristinamycin biosynthesis, the S. pristinaespiralis WT was grown in pristinamycin production medium and $2 \mathrm{mM}$ BL was added to the culture at the expected exponential growth phase at time point $12 \mathrm{~h}$. Cell culture samples were harvested at different time points $(12,24,48,72$, and $96 \mathrm{~h}$ ) and treated for pristinamycin extraction and analysis by HPLC. S. pristinaespiralis WT samples without the addition of BL served as a reference. HPLC analysis revealed a clearly increased pristinamycin production profile for the BL-induced samples in comparison to the non-induced samples (Figure 3). At $48 \mathrm{~h}$, the BL induced strains produced $\sim 30 \%$ more IA (PIA) $(0.13 \mathrm{mg} / \mathrm{ml})$ and $\sim 40 \%$ pristinamycin IIA (PIIA) $(0.21 \mathrm{mg} / \mathrm{ml})$ than the non-induced strains $(0.09 \mathrm{mg} / \mathrm{ml}$ PIA and $0.12 \mathrm{mg} / \mathrm{ml}$ PIIA, respectively) (Figure 3). Thus, it could be shown that the addition of synthetic BL induces pristinamycin production resulting in higher concentrations of pristinamycin.

\section{Pristinamycin Is Not an Effector for SpbR, PapR3, and PapR5}

Since addition of synthetic BL prevented formation of the GBLR-DNA interactions in EMSAs as mentioned above, it 
A<smiles>O=C1CCCO1</smiles>

C

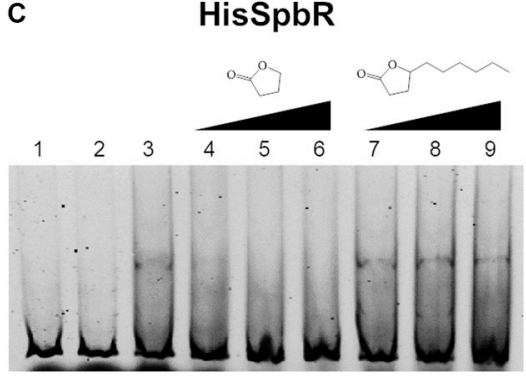

PpapR5
B<smiles>CCCCCCC1CCC(=O)O1</smiles>

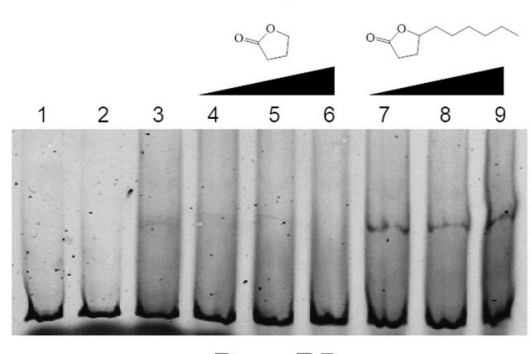

PpapR5
HisPapR5

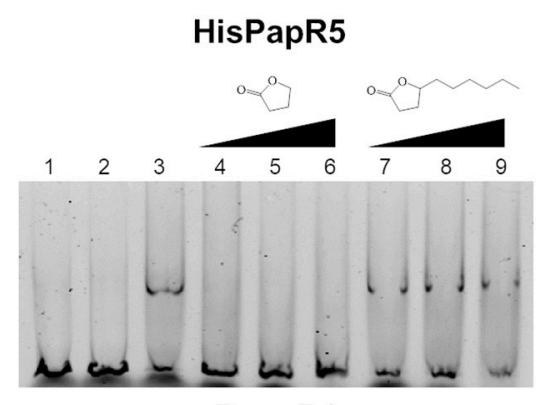

PpapR1

FIGURE 2 | Chemical structure of BL (A) and GDL (B). EMSAs (C) performed in 5\% acrylamide gel with cell lysate samples from SLspbR-OE (left), SLpapR3-OE (middle) with PpapR5 and SLpapR5-OE (right) with PpapR1 in the presence of synthetic GBLs. 1, promotor DNA; 2, promotor DNA + SLpGM190 lysate; 3 , promotor DNA + cell lysate from GBLR overexpression sample; 4-6, promotor DNA + cell lysate from GBLR overexpression sample + increasing concentration (1, 2, $4 \mu \mathrm{l}$, respectively) of BL; 7-9, promotor DNA + cell lysate from GBLR overexpression sample + increasing concentration (1, 2, $4 \mu$, respectively) of GDL.
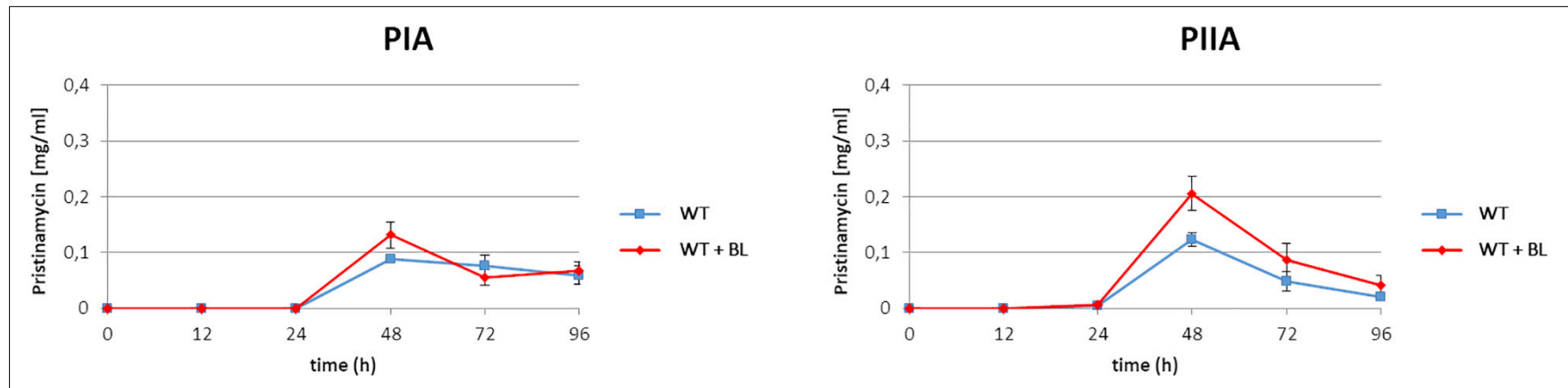

FIGURE 3 | Pristinamycin PIA and PIIA production process of S. pristinaespiralis WT with (red) and without addition of BL (blue). Production curves are shown as the averages of three different experiments conducted in triplicate. Error bars indicate standard deviations.

can be speculated that SpbR, PapR3, and PapR5 act as "real" GBLRs in S. pristinaespiralis. To find out whether proteinDNA interaction is influenced by pristinamycin as a potential effector molecule, EMSAs were carried out in the presence of increasing concentrations $(1,2,4,6 \mu \mathrm{l})$ of pure pristinamycin $(50 \mathrm{mg} / \mathrm{ml})$. Pristinamycin was added as a mixture of PIA and PIIA (1:1) dissolved in a solution of $1: 1$ methanol $\left(\mathrm{CH}_{3} \mathrm{OH}\right)$, dichloromethane $\left(\mathrm{CH}_{2} \mathrm{Cl}_{2}\right)$. Comparable concentrations of a 1:1 methanol/dichloromethane solution were added to each EMSA approach as negative control. EMSA assays showed that addition of pristinamycin did not affect the GBLR-DNA binding pattern (Figure 4, lines 3-6). Only samples with 4-6 $\mu$ l pristinamycin prevented the formation of a shifted band (Figure 4, lines $5,6)$, however, this was due to high concentrations of the methanol/dichloromethane solution since also no shifted band was observed in the corresponding negative control samples (Figure 4, lines 9, 10). These data showed that pristinamycin does not serve as an effector molecule for the GBLR-like regulators
SpbR, PapR3, and PapR5 and indicate that the pristinamycinspecific GBLRs do not function as pGBLRs.

\section{S. pristinaespiralis Culture Extracts Contain Endogenous Component(s) That Affect the GBLR-Like Regulator Binding}

The above mentioned EMSAs suggest that synthetic BL but not pristinamycin is able to bind as ligand to the pristinamycinspecific GBLR-like regulators. To investigate if the pristinamycinproducing strain $S$. pristinaespiralis owns components which affect the regulator-DNA binding activity of SpbR, PapR3, and PapR5, EMSAs were performed in the presence of S. pristinaespiralis culture extracts. For this purpose, the S. pristinaespiralis wild-type (WT) was grown in pristinamycin production medium and culture supernatant samples were harvested at different time points (30, 36, 42, 48 h). Cell culture samples were extracted and methanolic culture extracts were analyzed by HPLC for pristinamycin production. The different 


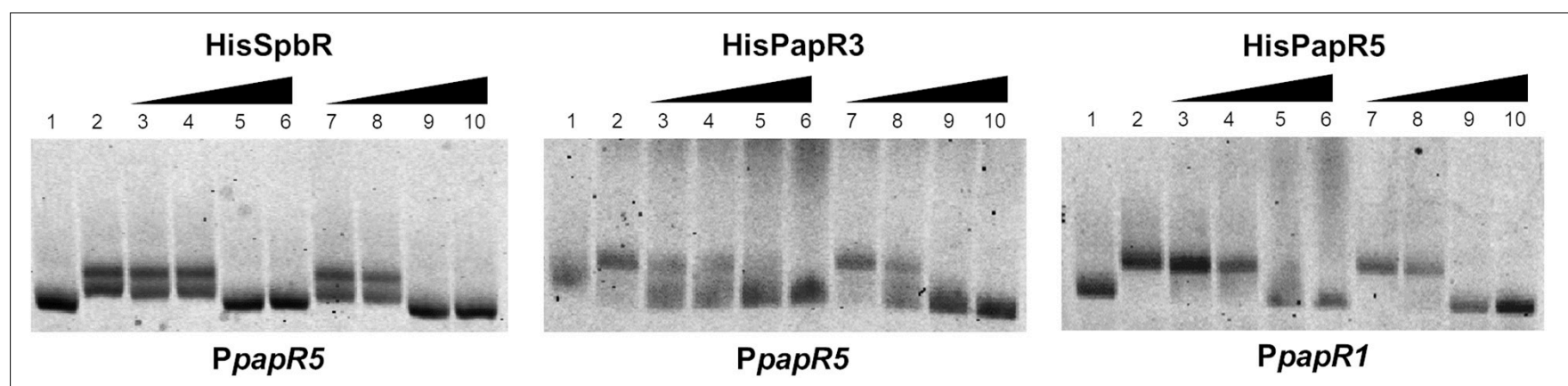

FIGURE 4 | EMSAs performed in 2\% agarose gel with cell lysate samples from SLspbR-OE (left), SLpapR3-OE (middle) with PpapR5 and SLpapR5-OE (right) with PpapR1 in the presence of pristinamycin. 1, promotor DNA; 2, promotor DNA + cell lysate from GBLR overexpression sample; 3-6, addition of increasing concentration (1, 2, 4, $6 \mu \mathrm{l})$ of pristinamycin; 7-10, addition of increasing concentration $(1,2,4,6 \mu l)$ of $\mathrm{CH}_{3} \mathrm{OH}_{\mathrm{H}} \mathrm{CH}_{2} \mathrm{Cl}_{2}(1: 1)$ as control.

cultivation time points were chosen to cover different phases of antibiotic production: before the start of antibiotic biosynthesis (30 h), during pristinamycin production $(36,42 \mathrm{~h})$ and at the maximum of pristinamycin production (48 h) (Mast et al., 2015). According to Paquet et al. (1992) effector production in S. pristinaespiralis begins at about $3 \mathrm{~h}$ before the initiation of pristinamycin production. Thus, at $36 \mathrm{~h}$ both, effector molecules and pristinamycin, should be present in the prepared extracts. In order to investigate whether endogenous S. pristinaespiralis factors bind to the regulatory proteins SpbR, PapR3, and PapR5, EMSAs were performed in the presence of $S$. pristinaespiralis WT extracts in a similar composition as described above. Data are shown for sample time point $36 \mathrm{~h}$ in Figure 5 and for residual cultivation time points in Supplementary Figure S2. Shifts with methanol served as negative control (Figure 5, lines 7-9). EMSA analysis showed that formation of a shifted band is prevented upon addition of 2-4 $\mu \mathrm{l}$ S. pristinaespiralis WT extract in samples with SpbR and PapR3 together with the promoter region PpapR5 and samples with PapR5 and PpapR1 (Figure 5, lines 4-6). Overall, these data exhibit that the $S$. pristinaespiralis WT culture extract contains endogenous components that serve as effectors for the pristinamycin-specific GBLR-like regulators SpbR, PapR3, and PapR5.

To investigate if the effectors, which cause the prevention of the regulator-DNA interaction are associated to the biosynthesis products of the pristinamycin pathway, EMSAs were performed in the presence of extract samples from the pristinamycindeficient mutant $S$. pristinaespiralis papR2::apra. In papR2::apra the essential SARP-type regulator gene $p a p R 2$ is deleted, which causes a complete loss of pristinamycin production (Mast et al., 2015; Supplementary Table S1). Strain cultivation, compound extraction and EMSA assays have been carried out in the same way as described for WT culture extract samples. EMSA assays revealed that addition of culture extracts from the papR2::apra mutant strain prevented the formation of shifted bands in the respective GBLR-regulator/DNA samples in a similar manner as observed for the WT extract samples [Supplementary Figure S6, lines 4-6 (36 h); Supplementary Figure S3 (30, $42,48 \mathrm{~h})$ ]. As pristinamycin-deficient culture extracts prevented shift formation, it can be concluded that also the papR2::apra mutant harbors the endogenous components that serve as ligands for the pristinamycin-specific GBLR-like regulators. This shows that pristinamycin does not act as functional ligand for SpbR, PapR3, and PapR5. This is to be expected since shifted bands would have been remained if pristinamycin served as a functional effector for the GBLR regulators, which however is absent in the papR2::apra mutant. This is in line with the observation that pure pristinamycin does not act as ligand for the pristinamycinspecific GBLR regulators and depicts that SpbR, PapR3, and PapR5 do not act as pGBLRs in S. pristinaespiralis.

\section{Identification of the Potential Pristinamycin Effector Biosynthesis Gene snbU}

EMSA assays showed that synthetic GBL but not pristinamycinassociated samples affect the DNA binding activity of SpbR, PapR3, and PapR5, which would hint for a GBL-like molecule as GBLR-interacting ligand in S. pristinaespiralis. So far, the chemical structure of the GBLR-interacting ligand molecule as well as its encoding biosynthetic gene(s) are unknown for $S$. pristinaespiralis. Well-known GBL biosynthesis genes are afs $A$ and $s c b A$ from $S$. griseus and S. coelicolor, respectively. Both genes encode for fatty acid synthase-like enzymes, whereby AfsA is responsible for the biosynthesis of the A-factor in S. griseus (Ando et al., 1997) and ScbA catalyzes the synthesis of the SCBs in S. coelicolor (Takano et al., 2008). No afs $A / s c b A$-like gene could be identified for $S$. pristinaespiralis (Mast et al., 2015). In S. pristinaespiralis the GBLR-like genes $s p b R$, $p a p R 3$, and $p a p R 5$ are part of a regulatory region localized at the right border of the pristinamycin biosynthesis gene cluster (Mast et al., 2011). Between the genes $p a p R 5$ and $s p b R$ the gene $s n b U$ is located, which encodes for a putative cytochrome P450 monooxygenase SnbU (Mast et al., 2015; Figure 7). SnbU shows amino acid sequence similarity to Orf16* (64\% identity, $75 \%$ similarity) from the tylosin producer Streptomyces fradiae and Cyp17 (63\% identity, $74 \%$ similarity) from the avermectin producer Streptomyces avermitilis. Both proteins have been suggested to be involved in effector biosynthesis in the respective antibiotic producers (Bignell et al., 2007; Kitani et al., 2011). In S. fradiae orf $16^{*}$ is located upstream of the gene $t y l P$ encoding the tylosin GBLR regulator TylP and orf $18^{*}$ encoding a putative acyl-CoA 


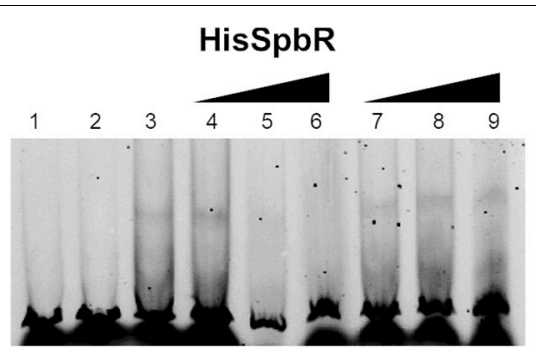

PpapR5

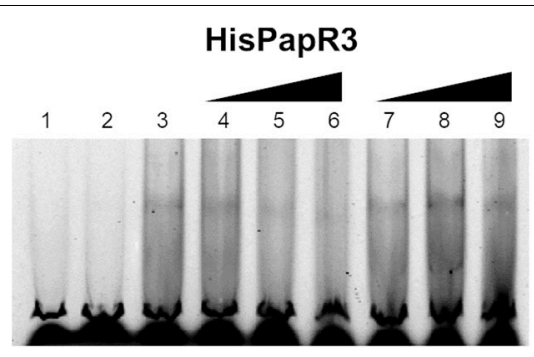

PpapR5

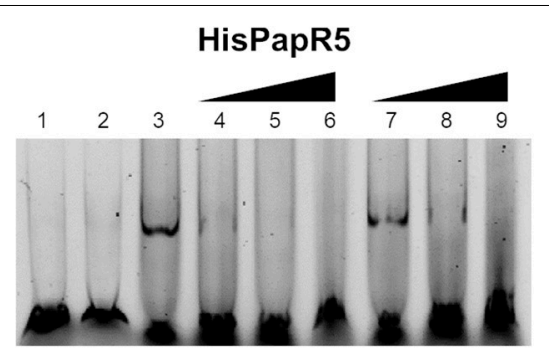

PpapR1

FIGURE 5 | EMSAs performed in 5\% acrylamide gel with cell lysate samples from SLspbR-OE (left), SLpapR3-OE (middle) with PpapR5 and SLpapR5-OE (right) with PpapR1 in the presence of $S$. pristinaespiralis WT culture extract. 1, promotor DNA; 2, promotor DNA + SLPGM190 lysate; 3 , promotor DNA + cell lysate from GBLR overexpression sample; 4-6, addition of increasing concentration (1, 2, $4 \mu \mathrm{l}$ ) of S. pristinaespiralis WT culture extract (36 h); 7-9, addition of increasing concentration $(1,2,4 \mu \mathrm{l})$ of methanol as control.

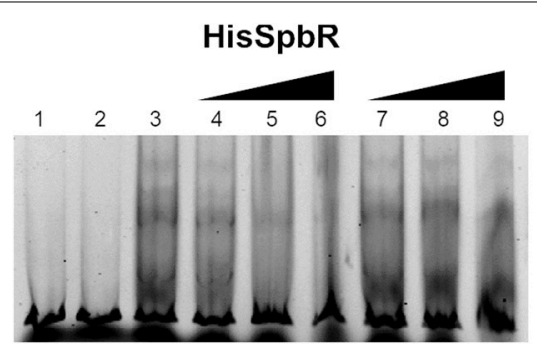

PpapR5

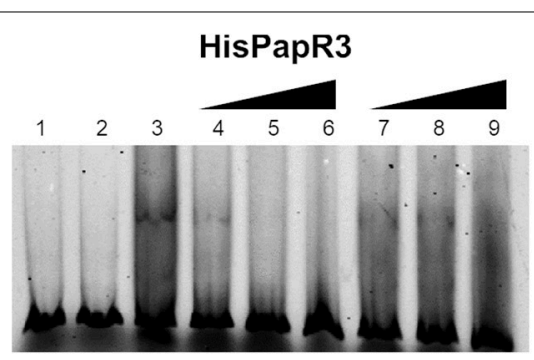

PpapR5

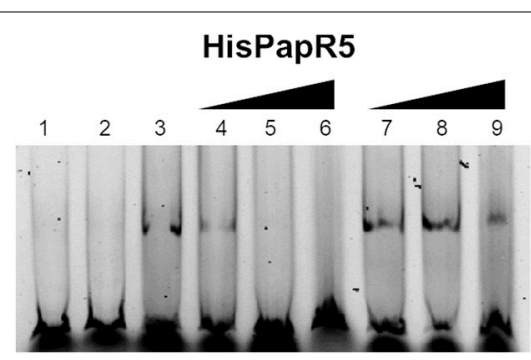

PpapR1

FIGURE 6 | EMSAs performed in 5\% acrylamide gel with cell lysate samples from SLspbR-OE (left), SLpapR3-OE (middle) with PpapR5 and SLpapR5-OE (right) with PpapR1 in the presence of S. pristinaespiralis papR2::apra culture extract. 1, promotor DNA; 2, promoter + SLpGM190 lysate; 3, promotor DNA + cell lysate from GBLR overexpression sample; 4-6, addition of increasing concentration (1, 2, 4 l) of S. pristinaespiralis papR2::apra culture extract (36 h); 7-9, addition of increasing concentration $(1,2,4 \mu \mathrm{l})$ of methanol as control.

oxidase (Figure 7). In S. avermitilis cyp17 shows a similar genetic localization and is found upstream of the gene avaR1 encoding the GBLR regulator AvaR1, and the gene aco encoding an acylCoA oxidase involved in avenolide effector synthesis (Kitani et al., 2011; Figure 7). Due to the amino acid sequence similarities between Orf16* and Cyp17 with SnbU and the comparable genetic organization of the coding genes within the respective antibiotic biosynthetic gene clusters, it can be assumed that SnbU is involved in the biosynthesis of the GBLR-interacting ligand(s) in $S$. pristinaespiralis. Thus, we analyzed the $s n b U$ gene in respect to its influence on pristinamycin production and GBLR-DNA interactions (see below).

\section{Inactivation of snbU Has Regulatory Influence on Pristinamycin Production}

In order to investigate the function of the putative effector biosynthesis gene $s n b U$, the gene was inactivated by insertion of an apramycin resistance cassette $\left(\mathrm{Apr}^{\mathrm{R}}\right)$ and the pristinamycin production of the resulting $s n b U:: a p r a$ mutant (Supplementary Table S1) was compared with that of the S. pristinaespiralis WT. For this purpose, the snbU::apra mutant and the WT were grown in pristinamycin production medium. Samples were taken at different time points $(24,48,72$, and $96 \mathrm{~h}$ ) and pristinamycin extracts were analyzed by HPLC. The HPLC analysis showed that PIA and PIIA production was increased in the snbU::apra mutant in comparison to the WT. At the production maximum at $48 \mathrm{~h}$, the snbU::apra mutant produced twice as much PIA $(0.19 \mathrm{mg} / \mathrm{ml})$ and $\sim 40 \%$ more PIIA $(0.21 \mathrm{mg} / \mathrm{ml})$ than the WT $(0.09 \mathrm{mg} / \mathrm{ml}$ PIA and $0.12 \mathrm{mg} / \mathrm{ml}$ PIIA, respectively) (Figure 8). Complementation of snbU::apra with the $s n b U$ expression construct pGM190/snbU

Streptomyces avermitilis
(Avermectin producer)
Streptomyces fradiae
(Tylosin producer)
Streptomyces pristinaespiralis
(Pristinamycin producer)
FIGURE 7 I Schematic presentation of the organization of regulatory genes in
the avermectin producer S. avermitilis (top), the tylosin producer S. fradiae
(middle) (Kitani et al., 2011), and the pristinamycin producer
S. pristinaespiralis (bottom) (modified according to Kitani et al., 2011).
Homologous genes are labeled with the same color: GBLR gene (red),
cytochrome P450 gene (green), and acyl-CoA oxidase gene (blue).




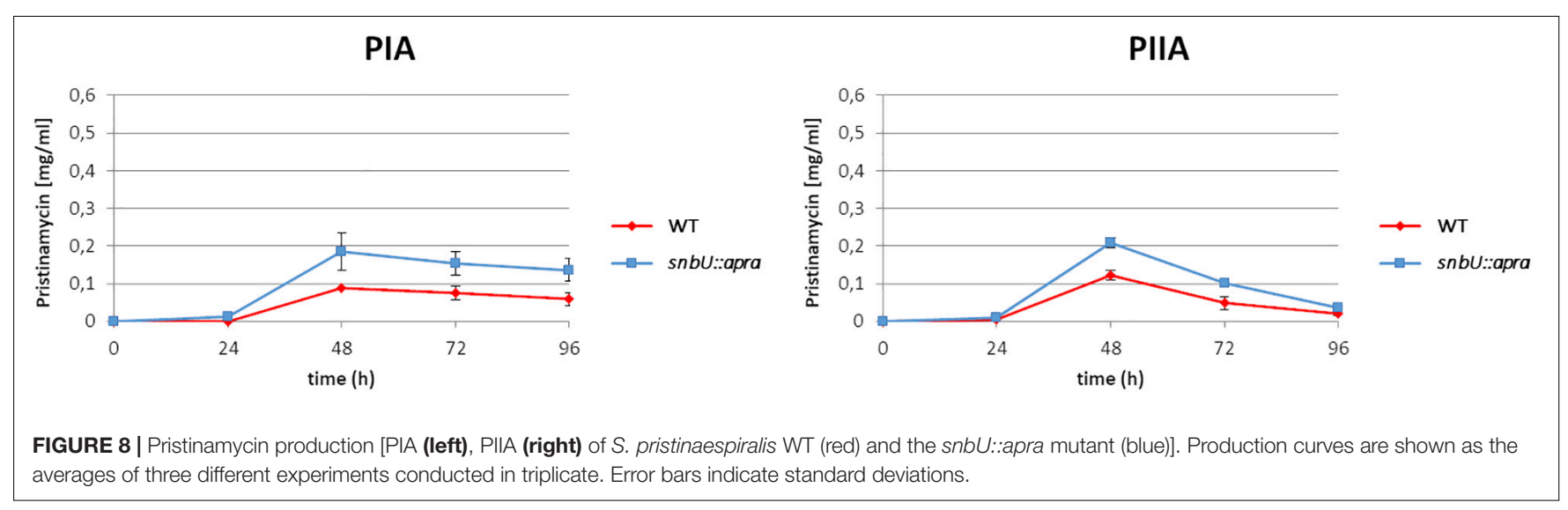

resulted in a pristinamycin production profile as similarly, observed for the WT strain (Supplementary Figure S4). These data show that inactivation of $s n b U$ results in an increased pristinamycin production. Thus, it can be concluded that SnbU has a regulatory influence on pristinamycin biosynthesis in S. pristinaespiralis.

\section{Inactivation of snbU Prevents Production of GBLR-Binding Endogenous Components}

In the above mentioned experiments it has been shown that S. pristinaespiralis culture extracts contain endogenous effectors that abolish the regulatory binding of the pristinamycinspecific GBLRs to their target promoters. Furthermore, it has been displayed that SnbU has a regulatory influence on pristinamycin production. To analyze if $s n b U$ is involved in the biosynthesis of the GBLR-interacting ligand EMSAs were performed with SpbR, PapR3, and PapR5 in the presence of culture extract samples from the $S$. pristinaespiralis snbU::apra mutant. EMSAs were carried out as mentioned above and are shown representatively for snbU::apra culture sample time point $36 \mathrm{~h}$. EMSA analysis showed that addition of culture extracts from the snbU::apra mutant did not prevent shift formation in any of the tested GBLR-promoter samples [Figure 9, lines 4-6 (36); Supplementary Figure S5 (30, 42, 48 h)]. This is different from the observation obtained from EMSAs in the presence of $S$. pristinaespiralis WT and papR2::apra mutant culture extracts, which did prevent shift formation (Figure 9 vs. Figures 5, 6, respectively). This finding shows that culture extracts from $S$. pristinaespiralis WT and papR2::apra contain endogenous factors that function as ligands for the pristinamycin-specific GBLR regulators, which are absent in the snbU::apra mutant. Thus, it can be concluded that SnbU is involved in the biosynthesis of the GBLR-interacting ligand molecule in S. pristinaespiralis.

\section{DISCUSSION}

Antibiotic biosynthesis is known to be governed by quorum sensing-like systems involving GBLs as signaling molecules.
Nodal points of regulation are represented by GBLR regulators, which sense the abundance of GBLs and transmit the effector signal to the biosynthesis output. In addition to the wellknown "real" GBLRs, which bind GBLs as ligands there are also several examples of "pseudo" GBLRs binding antibiotics or antibiotic intermediates as ligands (Biarnes-Carrera et al., 2015; Niu et al., 2016; Table 1). In S. pristinaespiralis three GBLR-like regulators are known to be involved in regulation of pristinamycin biosynthesis (Mast et al., 2015). So far, it was not known if the regulators act as real GBLRs or pGBLRs. To elucidate the function of the respective GBLR-like regulators in $S$. pristinaespiralis, EMSAs were carried out in this study, which showed that all three pristinamycin-specific GBLR-like regulators, SpbR, PapR3, and PapR5, belong to the group of "real" GBLRs since regulator-DNA interactions were influenced by GBL-associated samples but not by pristinamycinspecific samples.

It remains to be elucidated if all three GBLRs, SpbR, PapR3, and PapR5, sense the same chemical ligand or if structurally different effector molecules are recognized and how this translates to the working model of the pristinamycin hierarchical signaling cascade. Additional information on the pristinamycin-specific GBLRs has been gained by bioinformatic analysis with the I-TASSER software, which allows to perform in silico protein structure and function predictions (Yang et al., 2014). I-TASSER enables to predict three-dimensional structural models and potential ligands of protein molecules based on amino acid sequences. I-TASSER analysis with the amino acid sequences of SpbR, PapR3, and PapR5 revealed nucleic acids as a potential ligand for all three GBLR-like proteins (Supplementary Figure S6 and Supplementary Table S2). Furthermore, small, unpolar, mostly aromatic-like metabolites have been identified as potential ligands (Supplementary Figure S6 and Supplementary Table S2), whereby SpbR and PapR5 showed more overlap in the suggested ligand types than PapR3 (Supplementary Table S2). This may indicate that SpbR and PapR5 sense the same or a similar ligand substance, which is different from the effector molecule recognized by PapR3. The fact that no GBL- or avenolide-like molecule has been proposed by the I-TASSER program as potential ligand might be because no GBLRs from antibiotic-producing 


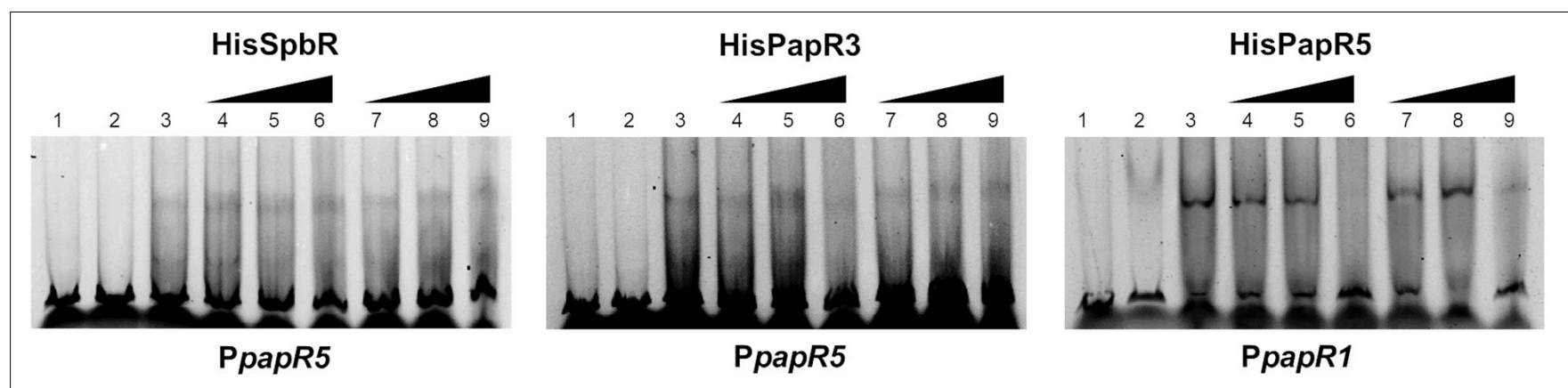

FIGURE 9 | EMSAs performed in 5\% acrylamide gel with cell lysate samples from SLspbR-OE (left), SLpapR3-OE (middle) with PpapR5 and SLpapR5-OE (right) with PpapR1 in the presence of $S$. pristinaespiralis snbU::apra culture extract. 1, promotor DNA; 2, promotor DNA + SLpGM190 lysate; 3 , promotor DNA + cell lysate from GBLR overexpression sample; 4-6, addition of increasing concentration (1, 2, 4 l) of S. pristinaespiralis snbU::apra culture extract (36 h); 7-9, addition of increasing concentration $(1,2,4 \mu \mathrm{l})$ of methanol as control.

actinomycetes with their cognate ligands are deposited in the I-TASSER data base.

The structure of the pristinamycin-specific GBLR-interacting ligand molecule(s) as well as the encoding biosynthetic gene(s) are unknown for $S$. pristinaespiralis so far. In this work, the gene snbU, which codes for a putative cytochrome P450 monooxygenase is described to be involved in the biosynthesis of the pristinamycin-specific GBLR-interacting ligand(s). Surprisingly, inactivation of $s n b U$ in S. pristinaespiralis led to an increased pristinamycin production profile. In contrast, inactivation of genes involved in antibiotic effector biosynthesis in other streptomycetes, often result in loss of antibiotic production or a decrease in production yields. For example, inactivation of $a f s A$, which is responsible for A-factor biosynthesis in $S$. griseus, abolished streptomycin production completely (Horinouchi, 2002). However, also antibiotic overproduction has been reported for producers with inactivated GBL biosynthesis genes. For example, in S. coelicolor, the deletion of the GBL synthase gene $s c b A$, which abolished the biosynthesis of the SCB1 effector molecule resulted in precocious and increased production of ACT and RED (Takano et al., 2008). The cognate GBLR for the SCB1 effector in S. coelicolor is ScbR, which represses its own transcription, as well as that of $s c b A$. For $S$. coelicolor it has been proposed that deletion of $s c b A$ abolishes SCB1 production, which results in a basal level of ligand-free ScbR, which itself is suggested to repress the synthesis of a hierarchical subordinate transcriptional repressor of antibiotic biosynthesis giving rise to overproduction of ACT and RED (Takano et al., 2008). Similarly, the pristinamycin overproducing effect of the snbU::apra mutant might be explained by the complex hierarchical organization of the regulatory signaling cascade. In this respect inactivation of $s n b U$ would cause loss of effector synthesis resulting in low levels of ligand-free GBLR, e.g., SpbR, which would be inefficient for repressing the transcription of the hierarchical subordinate repressor gene papR5, resulting in increased pristinamycin production levels. This would be in accordance with the observation that deletion of papR5 in S. pristinaespiralis results in dramatically increased pristinamycin production (Mast et al., 2015). Cytochrome P450 monooxygenases have been suggested before to be involved in antibiotic effector synthesis. In $S$. fradiae and $S$. avermitilis, orf $16^{*}$ and cyp 17 both encode for predicted cytochrome P450 monooxygenases, respectively, and are suggested to participate in effector synthesis in the respective antibiotic producers. $s n b U$, orf $16^{*}$ and cyp 17 are each located downstream of a GBLRtype regulator gene and a gene encoding an acyl-CoA oxidase (Figure 7). Also, the acyl-CoA oxidase encoding genes orf $18^{*}$ and aco from S. fradiae and S. avermitilis, respectively, have been reported to be involved in effector biosynthesis, whereas the encoded GBLR proteins serve as the ligand receptors. Inactivation of $\operatorname{orfl}^{*}$ and orf $^{*} 18$ in $S$. fradiae resulted in a reduction of tylosin production to $40-60 \%$ of the WT production level and loss of production of the TylP-binding ligand (Bignell et al., 2007). Thus, it was concluded that both genes are involved in the synthesis of the TylP-associated ligand. However, so far the TylP ligand has not been identified and the involvement of orf $16^{*}$ and orf $18^{*}$ in ligand synthesis has not been demonstrated (Zou et al., 2014). Inactivation of aco in S. avermitilis resulted in a reduction of avermectin production to $\sim 6 \%$ of wildtype levels and loss of avenolide effector synthesis (Kitani et al., 2011). Aco has been proposed to introduce the double bond into the C2-C3 position of the avenolide effector from S. avermitilis (Kitani et al., 2011; Supplementary Figure S7). A putative aco-homologous gene, snbX (accession number: MT563328), has been identified downstream of $s p b R$ in the course of preparing this manuscript and is not experimental part of this study (Figure 7). The gene was identified based on a newly obtained PacBio genome sequence from S. pristinaespiralis Pr11 (accession number CP059696). The predicted gene product SnbX shows homology to the putative acyl-CoA oxidases Orf18 (46\% identity, 58\% similarity) from S. fradiae and Aco (53\% identity, 64\% similarity) from $\mathrm{S}$. avermitilis and thus may also be involved in the biosynthesis of the GBLR-interacting ligand(s) in S. pristinaespiralis. Due to the genetic similarity of the mentioned effector synthesis genes and the experimental data on the avenolide effector, it can be expected that the pristinamycin-specific ligand(s) may represent a signaling molecule of the avenolide type. This is also in line with the observation that alkaline treatment of GBLRsamples in the presence of $S$. pristinaespiralis WT extracts did 
not influence the shift performance (Supplementary Figure S8), as similarly, observed for respective samples with the avenolide effector (Kitani et al., 2011). Instead, GBLs are known to be sensitive to alkaline treatment but resistant to treatments with protease, heat and acid ( $\mathrm{Du}$ et al., 2011). Thus, also these data support the hypothesis that the signaling molecule from S. pristinaespiralis belongs to the avenolide type of autoregulators. So far, it cannot be excluded that additional genes/enzymes are involved in effector synthesis in S. pristinaespiralis. The pristinamycin gene region contains three genes of unknown function - snbS, snbW, and $s n b V$. The genes are located at the right border of the cluster next to the "regulatory operon" upstream of papR3 (Mast et al., 2011). snbS encodes a putative methylmalonyl-CoA decarboxylase, while $s n b W$ and $s n b V$ carry the information for hypothetical proteins. In order to investigate their involvement in pristinamycin biosynthesis and/or regulation $s n b S$, $s n b W$, and $s n b V$ will be inactivated in ongoing experiments. Furthermore, it would be important to isolate and solve the chemical structure of the GBLR-interacting molecule from S. pristinaespiralis, which so far was hindered by low ligand concentrations. However, the availability of a ligand-free $s n b U:: a p r a$ mutant, which can serve as a reference in analytic analysis, may facilitate structure elucidation approaches in future studies.

In this work, we demonstrated that SpbR, PapR3, and PapR5 belong to the class of real GBLRs, which most likely bind avenolide-type effector molecules as ligands. The gene snbU encoding a predicted cytochrome $\mathrm{P} 450$ monooxygenase has been demonstrated to be involved in the biosynthesis of the GBLRinteracting ligand.

\section{MATERIALS AND METHODS}

\section{Bacterial Strains, Plasmids, and Cultivation Conditions}

The bacterial strains and plasmids used in this study are listed in Supplementary Table S1. For routine cloning strategies, Escherichia coli NovaBlue (Novagen) was used. E. coli strains were grown in Luria-Bertani (LB) medium (Maniatis et al., $1989)$ at $37^{\circ} \mathrm{C}$, supplemented with kanamycin, apramycin, or ampicillin $(50,100$, or $150 \mu \mathrm{g} / \mathrm{ml}$, respectively) when appropriate. S. pristinaespiralis Pr11 (Aventis Pharma) was used for the generation of the $s n b U$ insertion mutant. For cultivation and harvesting of genomic DNA, Streptomyces strains were grown in $100 \mathrm{ml}$ of S-medium (Kieser et al., 2000) in $500 \mathrm{ml}$ Erlenmeyer flasks (with steel springs) on an orbital shaker $(180 \mathrm{rpm})$ at $28^{\circ} \mathrm{C}$. Kanamycin and apramycin (50 and 100 $\mu \mathrm{g} / \mathrm{ml}$, respectively) were added to liquid cultures when required. For pristinamycin production analysis, S. pristinaespiralis strains were cultivated as reported previously (Mast et al., 2011). For EMSA analysis, the $S$. pristinaespiralis strains were sampled at different time points after 30, 36, 42, and 48 h. $100 \mathrm{ml}$ of S. pristinaespiralis culture was extracted with $100 \mathrm{ml}$ ethyl acetate for $20 \mathrm{~min}$ and concentrated completely in vacuo. The extract was then redissolved in appropriate volumes of methanol ( $2 \mathrm{ml}$ methanol for $100 \mathrm{ml}$ extraction volume). Streptomyces lividans T7 (Fischer, 1996; Supplementary Table S1) was used for protein expression experiments. S. lividans strains were GBLR expression strains SLpapR3-OE, SLpapR5-OE, SLspbR-OE, and SLPGM190 (control) were cultivated in yeast extract-malt extract (YEME) medium as reported before (Mast et al., 2015).

\section{Molecular Cloning}

DNA manipulation procedures were performed as described previously for E. coli (Maniatis et al., 1989) and for Streptomyces (Kieser et al., 2000). The primers used for PCR were obtained from Eurofins MWG Biotech AG (Ebersberg, Germany) and are listed in Supplementary Table S1.

\section{Expression of the Pristinamycin Regulators}

For protein expression, S. lividans GBLR expression strains SLpapR3-OE, SLpapR5-OE, SLspbR-OE, and SLpGM190 (control) were constructed and cultivated as reported before (Mast, 2008; Mast et al., 2015; Supplementary Table S1).

\section{Electromobility Shift Assays (EMSAs)}

DNA fragments of the upstream regions of the regulatory genes papR1 (PpapR1, $240 \mathrm{bp}$ ) and papR5 (PpapR5, $260 \mathrm{bp}$ ) were amplified by PCR from genomic DNA of $S$. pristinaespiralis Pr11 with primers listed in Supplementary Table S1. Promoter DNA amplificates included a 16 bp Cy5 adapter sequence, each at the $3^{\prime}$ and $5^{\prime}$ end, which was added via the respective primer sequences. The generated amplificates were used as templates in a second PCR approach together with a Cy5 primer (Supplementary Table S1) in order to conduct Cy5 labeling of the promoter regions. For EMSAs, cell lysates from S. lividans strains were used, which overexpressed the respective His-tagged GBLR-like regulators together with $2 \mathrm{ng}$ of the respective Cy5-labeled promotor region, PpapR5 or PpapR1.

DNA-regulator binding reactions were performed at room temperature as described previously (Mast et al., 2015). The samples were loaded onto a $2 \%$ agarose gel for testing the specificity of regulator-DNA binding and effect of pristinamycin addition. For EMSAs with synthetic GBLs and S. pristinaespiralis culture extracts 5\% acrylamide gels were used. EMSAs were carried out with variable concentrations of regulator lysate sample as reported before (Mast et al., 2015). To verify the specificity of the regulator-DNA binding, an excess of unlabeled, specific target DNA (PpapR1 or PpapR5) and nonspecific DNA $(h r d B)$, respectively, was added to the EMSA mixture as described previously (Mast et al., 2015). DNA bands were visualized by fluorescence imaging using a Typhoon Trio $^{\mathrm{TM}}$ Variable Mode Imager (GE Healthcare). All EMSAs were carried out at least three times independently. Pure BL $(0.948 \mathrm{~g} / \mathrm{ml})$ and GDL $(1.12 \mathrm{~g} / \mathrm{ml})$ were purchased by Sigma-Aldrich. Pure pristinamycin $(50 \mathrm{mg} / \mathrm{ml})$, consisting of a 1:1 mixture of PIA and PIIA was obtained from SanofiAventis Pharma. 


\section{Construction of the S. pristinaespiralis snbU::apra Mutant}

For construction of a snbU apramycin insertion mutant, a $\sim 2.6$ $\mathrm{kb}$ fragment was amplified by PCR using Taq polymerase and primers snbUfw/snbUrev listed in Supplementary Table S1. Cosmid 1/12 DNA served as template, which was obtained from a $S$. pristinaespiralis cosmid library and harbored part of the pristinamycin gene cluster. The PCR-derived $\sim 2.6 \mathrm{~kb}$ amplificate $s n b U^{\prime}$ included the 1,320 bp snbU coding sequence, as well as a 437 bp up- and a 787 bp downstream region. $s n b U^{\prime}$ was subcloned into the EcoRV-linearized E. coli cloning vector pDrive, resulting in the construct pDrive/snbU.' snbU' was isolated from pDrive/snbU' as EcoRI fragment and ligated to the EcoRI-restricted E. coli vector pK18 (Pridmore, 1987; Supplementary Table S1), resulting in the construct pK18/snbU.' A $1.5 \mathrm{~kb}$ apramycin resistance cassette $\left(\mathrm{Apr}^{\mathrm{R}}\right)$ was isolated as an EcoRV/SmaI fragment from pEH13 (Heinzelmann et al., 2001; Supplementary Table S1) and cloned into the NruI restriction site of snbU,' which yielded the final mutagenesis construct pK18/snbUapra, in which the gene $s n b U$ is inactivated by an apramycin resistance cassette. This construct was transferred to S. pristinaespiralis Pr11 by protoplast transformation, followed by a selection for apramycin-resistant and kanamycin-sensitive transformants, resulting in the $S$. pristinaespiralis mutant snbU::apra. The snbU mutant was complemented by introducing the expression plasmid pGM190/snbU to snbU::apra, resulting in strain snbU::apra pGM190/snbU (Supplementary Table S1).

\section{Pristinamycin Analysis}

Pristinamycin was detected by HPLC analysis as reported before (Mast et al., 2011).

\section{Bioinformatic Analysis}

In silico protein structure analysis was performed with the I-TASSER software ${ }^{1}$ (Yang et al., 2014) and the amino acid sequences of SpbR, PapR3, and PapR5 (Mast et al., 2011).

${ }^{1}$ https://zhanglab.ccmb.med.umich.edu/I-TASSER/

\section{REFERENCES}

Ando, N., Matsumori, N., Sakuda, S., Beppu, T., and Horinouchi, S. (1997). Involvement of afsA in A-factor biosynthesis as a key enzyme. J. Antibiot. 50, 847-852. doi: 10.7164/antibiotics.50.847

Arakawa, K., Tsuda, N., Taniguchi, A., and Kinashi, H. (2012). The butenolide signaling molecules SRB1 and SRB2 Induce lankacidin and lankamycin production in Streptomyces rochei. Chembiochem 13, 1447-1457. doi: 10.1002/ cbic. 201200149

Biarnes-Carrera, M., Breitling, R., and Takano, E. (2015). Butyrolactone signalling circuits for synthetic biology. Curr. Opin. Chem. Biol. 28, 91-98. doi: 10.1016/J. CBPA.2015.06.024

Bibb, M. J. (2005). Regulation of secondary metabolism in streptomycetes. Curr. Opin. Microbiol. 8, 208-215. doi: 10.1016/J.MIB.2005. 02.016

Bignell, D. R. D., Bate, N., and Cundliffe, E. (2007). Regulation of tylosin production: role of a TylP-interactive ligand. Mol. Microbiol. 63, 838-847. doi: 10.1111/j.1365-2958.2006.05541.x

\section{DATA AVAILABILITY STATEMENT}

The datasets presented in this study can be found in online repositories. The names of the repository/repositories and accession number(s) can be found in the article/Supplementary Material.

\section{AUTHOR CONTRIBUTIONS}

FH carried out the EMSA assays and pristinamycin production analysis. AK and FH did the HPLC analyses. YM carried out bioinformatic analyses and designed, supervised, and coordinated the study. FH and YM wrote the manuscript. All authors contributed to the article and approved the submitted version.

\section{FUNDING}

We acknowledge the funding received from the German Center for Infection Research (DZIF), project TTU 09.819 and a grant from the Athene-Programm für Nachwuchswissenschaftlerinnen of the University of Tübingen given to YM.

\section{ACKNOWLEDGMENTS}

We thank Regina Ort-Winklbauer and Michael Fischer from University of Tübingen for excellent technical assistance and W. Wohlleben for helpful discussions during the project. We gratefully acknowledge the provision of purified pristinamycin from Sanofi Aventis Pharma.

\section{SUPPLEMENTARY MATERIAL}

The Supplementary Material for this article can be found online at: https://www.frontiersin.org/articles/10.3389/fmicb. 2020.580990/full\#supplementary-material

Bunet, R., Song, L., Mendes, M. V., Corre, C., Hotel, L., Rouhier, N., et al. (2011). Characterization and manipulation of the pathway-specific late regulator AlpW reveals Streptomyces ambofaciens as a new producer of Kinamycins. J. Bacteriol. 193, 1142-1153. doi: 10.1128/jb.01269-10

Cuthbertson, L., and Nodwell, J. R. (2013). The TetR family of regulators. Microbiol. Mol. Biol. Rev. 77, 440-475. doi: 10.1128/mmbr.00018-13

Du, Y.-L., Shen, X.-L., Yu, P., Bai, L.-Q., and Li, Y.-Q. (2011). Gammabutyrolactone regulatory system of Streptomyces chattanoogensis links nutrient utilization, metabolism, and development. Appl. Environ. Microbiol. 77, 84158426. doi: 10.1128/aem.05898-11

Fischer, J. (1996). Entwicklung Eines Regulierbaren Expressionssystems zur Effizienten Synthese Rekombinanter Proteine in Streptomyces lividans. Ph.D. thesis, University of Stuttgart, Stuttgart.

Folcher, M., Gaillard, H., Nguyen, L. T., Nguyen, K. T., Lacroix, P., BamasJacques, N., et al. (2001). Pleiotropic functions of a Streptomyces pristinaespiralis autoregulator receptor in development, antibiotic biosynthesis, and expression of a superoxide dismutase. J. Biol. Chem. 276, 44297-44306. doi: 10.1074/jbc. M101109200 
Gao, X., Wang, Y., and Chu, J. (2019). A preliminary study on the impact of exogenous A-factor analogue 1,4-butyrolactone on stimulating bitespiramycin biosynthesis. Bioprocess Biosyst. Eng. 42, 1903-1913. doi: 10.1007/s00449-01902184-9

Heinzelmann, E., Kienzlen, G., Kaspar, S., Recktenwald, J., Wohlleben, W., and Schwartz, D. (2001). The phosphinomethylmalate isomerase gene pmi, encoding an aconitase-like enzyme, is involved in the synthesis of phosphinothricin tripeptide in Streptomyces viridochromogenes. Appl. Environ. Microbiol. 67, 3603-3609. doi: 10.1128/AEM.67.8.3603-3609. 2001

Horinouchi, S. (2002). A microbial hormone, A-factor, as a master switch for morphological differentiation and secondary metabolism in Streptomyces griseus. Front. Biosci. 7, 2045-2057.

Horinouchi, S. (2007). Mining and polishing of the treasure trove in the bacterial genus Streptomyces. Biosci. Biotechnol. Biochem. 71, 283-299. doi: 10.1271/bbb. 60627

Hsiao, N.-H., Soding, J., Linke, D., Lange, C., Hertweck, C., Wohlleben, W., et al. (2007). ScbA from Streptomyces coelicolor A3(2) has homology to fatty acid synthases and is able to synthesize -butyrolactones. Microbiology 153, 1394-1404. doi: 10.1099/mic.0.2006/004432-0

Kawachi, R., Akashi, T., Kamitani, Y., Sy, A., Wangchaisoonthorn, U., Nihira, T., et al. (2000). Identification of an AfsA homologue (BarX) from Streptomyces virginiae as a pleiotropic regulator controlling autoregulator biosynthesis, virginiamycin biosynthesis and virginiamycin M1 resistance. Mol. Microbiol. 36, 302-313. doi: 10.1046/j.1365-2958.2000.01819.x

Kieser, T., Bibb, M. J., Buttner, M. J., Chater, K. F., Hopwood, D. A., and John Innes Foundation (2000). Practical Streptomyces Genetics.

Kinoshita, H., Ipposhi, H., Okamoto, S., Nakano, H., Nihira, T., and Yamada, Y. (1997). Butyrolactone autoregulator receptor protein (BarA) as a transcriptional regulator in Streptomyces virginiae. J. Bacteriol. 179, 6986-6993. doi: 10.1128/JB.179.22.6986-6993.1997

Kitani, S., Hoshika, M., and Nihira, T. (2008). Disruption of $s s c R$ encoding a $\gamma$-butyrolactone autoregulator receptor in Streptomyces scabies NBRC 12914 affects production of secondary metabolites. Folia Microbiol. 53, 115-124. doi: 10.1007/s12223-008-0017-y

Kitani, S., Miyamoto, K. T., Takamatsu, S., Herawati, E., Iguchi, H., Nishitomi, K., et al. (2011). Avenolide, a Streptomyces hormone controlling antibiotic production in Streptomyces avermitilis. Proc. Natl. Acad. Sci. U.S.A. 108, 1641016415. doi: $10.1073 /$ pnas. 1113908108

Kurniawan, Y. N., Kitani, S., Iida, A., Maeda, A., Lycklama a Nijeholt, J., Lee, Y. J., et al. (2016). Regulation of production of the blue pigment indigoidine by the pseudo $\gamma$-butyrolactone receptor FarR2 in Streptomyces lavendulae FRI-5. J. Biosci. Bioeng. 121, 372-379. doi: 10.1016/J.JBIOSC.2015. 08.013

Lee, Y. J., Kitani, S., Kinoshita, H., and Nihira, T. (2008). Identification by gene deletion analysis of barS2, a gene involved in the biosynthesis of $\gamma$-butyrolactone autoregulator in Streptomyces virginiae. Arch. Microbiol. 189, 367-374. doi: 10.1007/s00203-007-0327-5

Lee, Y. J., Kitani, S., and Nihira, T. (2010). Null mutation analysis of an afsAfamily gene, barX, that is involved in biosynthesis of the $\gamma$-butyrolactone autoregulator in Streptomyces virginiae. Microbiology 156, 206-210. doi: 10. 1099/mic.0.032003-0

Liu, G., Chater, K. F., Chandra, G., Niu, G., and Tan, H. (2013). Molecular regulation of antibiotic biosynthesis in Streptomyces. Microbiol. Mol. Biol. Rev. 77, 112-143. doi: $10.1128 / \mathrm{mmbr} .00054-12$

Maniatis, T., Fritsch, E., and Sambrook, J. (1989). Molecular Cloning: a Laboratory Manual. Cold Spring Harbor, NY: Cold Spring Harbor Laboratory Press.

Mast, Y. (2008). Biosynthetische und Regulatorische Aspekte der PristinamycinProduktion in Streptomyces pristinaespiralis. Ph.D. thesis, Eberhard Karls University of Tübingen, Tübingen.

Mast, Y., Guezguez, J., Handel, F., and Schinko, E. (2015). A complex signaling cascade governs pristinamycin biosynthesis in Streptomyces pristinaespiralis. Appl. Environ. Microbiol. 81, 6621-6636. doi: 10.1128/aem.00 728-15

Mast, Y., Weber, T., Gölz, M., Ort-Winklbauer, R., Gondran, A., Wohlleben, W., et al. (2011). Characterization of the "pristinamycin supercluster" of Streptomyces pristinaespiralis. Microb. Biotechnol. 4, 192-206. doi: 10.1111/j. 1751-7915.2010.00213.x
Mast, Y., and Wohlleben, W. (2014). Streptogramins - two are better than one! Int. J. Med. Microbiol. 304, 44-50. doi: 10.1016/J.IJMM.2013.08.008

Matsuno, K., Yamada, Y., Lee, C.-K., and Nihira, T. (2004). Identification by gene deletion analysis of barB as a negative regulator controlling an early process of virginiamycin biosynthesis in Streptomyces virginiae. Arch. Microbiol. 181, 52-59. doi: 10.1007/s00203-003-0625-5

McCormick, J. R., and Flärdh, K. (2012). Signals and regulators that govern Streptomyces development. FEMS Microbiol. Rev. 36, 206-231. doi: 10.1111/j. 1574-6976.2011.00317.x

Mingyar, E., Feckova, L., Novakova, R., Bekeova, C., and Kormanec, J. (2015). A $\gamma$-butyrolactone autoregulator-receptor system involved in the regulation of auricin production in Streptomyces aureofaciens CCM 3239. Appl. Microbiol. Biotechnol. 99, 309-325. doi: 10.1007/s00253-014-6057-0

Nishida, H., Ohnishi, Y., Beppu, T., and Horinouchi, S. (2007). Evolution of $\gamma$-butyrolactone synthases and receptors in Streptomyces. Environ. Microbiol. 9, 1986-1994. doi: 10.1111/j.1462-2920.2007.01314.x

Niu, G., Chater, K. F., Tian, Y., Zhang, J., and Tan, H. (2016). Specialised metabolites regulating antibiotic biosynthesis in Streptomyces spp. FEMS Microbiol. Rev. 40, 554-573. doi: 10.1093/femsre/fuw012

Paquet, V., Goma, G., and Soucaille, P. (1992). Induction of pristinamycins production in Streptomyces pristinaespiralis. Biotechnol. Lett. 14, 1065-1070. doi: $10.1007 / \mathrm{BF} 01021060$

Pridmore, R. D. (1987). New and versatile cloning vectors with kanamycinresistance marker. Gene 56, 309-312. doi: 10.1016/0378-1119(87)90 149-1

Ramos, J. L., Martínez-Bueno, M., Molina-Henares, A. J., Terán, W., Watanabe, K., Zhang, X., et al. (2005). The TetR family of transcriptional repressors. Microbiol. Mol. Biol. Rev. 69, 326-356. doi: 10.1128/MMBR.69.2.326-356. 2005

Salehi-Najafabadi, Z., Barreiro, C., Rodríguez-García, A., Cruz, A., López, G. E., and Martín, J. F. (2014). The gamma-butyrolactone receptors BulR1 and BulR2 of Streptomyces tsukubaensis: tacrolimus (FK506) and butyrolactone synthetases production control. Appl. Microbiol. Biotechnol. 98, 4919-4936. doi: 10.1007/s00253-014-5595-9

Shikura, N., Yamamura, J., and Nihira, T. (2002). barS1, a gene for biosynthesis of a $\gamma$-butyrolactone autoregulator, a microbial signaling molecule eliciting antibiotic production in Streptomyces species. J. Bacteriol. 184, 5151-5157. doi: 10.1128/JB.184.18.5151-5157.2002

Takano, E., Chakraburtty, R., Nihira, T., Yamada, Y., and Bibb, M. J. (2008). A complex role for the $\gamma$-butyrolactone SCB1 in regulating antibiotic production in Streptomyces coelicolor A3(2). Mol. Microbiol. 41, 1015-1028. doi: 10.1046/j. 1365-2958.2001.02562.x

Takano, E., Nihira, T., Hara, Y., Jones, J. J., Gershater, C. J. L., Yamada, Y., et al. (2000). Purification and structural determination of SCB1, a $\gamma$-butyrolactone that elicits antibiotic production in Streptomyces coelicolor A3(2). J. Biol. Chem. 275, 11010-11016. doi: 10.1074/jbc.275.15.11010

Tan, G.-Y., Bai, L., and Zhong, J.-J. (2013). Exogenous 1,4-butyrolactone stimulates A-factor-like cascade and validamycin biosynthesis in Streptomyces hygroscopicus 5008. Biotechnol. Bioeng. 110, 2984-2993. doi: 10.1002/bit.2 4965

Tanaka, Y., and Omura, S. (1990). Metabolism and products of actinomycetes. An introduction. Actinomycetologica 4, 13-14. doi: 10.3209/saj.4_13

Wang, J., Wang, W., Wang, L., Zhang, G., Fan, K., Tan, H., et al. (2011). A novel role of 'pseudo' $\gamma$-butyrolactone receptors in controlling $\gamma$-butyrolactone biosynthesis in Streptomyces. Mol. Microbiol. 82, 236-250. doi: 10.1111/j.13652958.2011.07811.x

Wang, W., Zhang, J., Liu, X., Li, D., Li, Y., Tian, Y., et al. (2018). Identification of a butenolide signaling system that regulates nikkomycin biosynthesis in Streptomyces. J. Biol. Chem. 293, 20029-20040. doi: 10.1074/jbc.RA118.005667

Xu, G., Wang, J., Wang, L., Tian, X., Yang, H., Fan, K., et al. (2010). "Pseudo" $\gamma$-butyrolactone receptors respond to antibiotic signals to coordinate antibiotic biosynthesis. J. Biol. Chem. 285, 27440-27448. doi: 10.1074/JBC.M110.143081

$\mathrm{Xu}$, G., and Yang, S. (2019). Regulatory and evolutionary roles of pseudo $\gamma$-butyrolactone receptors in antibiotic biosynthesis and resistance. Appl. Microbiol. Biotechnol. 103, 9373-9378. doi: 10.1007/s00253-019-10219-0

Yang, J., Yan, R., Roy, A., Xu, D., Poisson, J., and Zhang, Y. (2014). The I-TASSER suite: protein structure and function prediction. Nat. Methods 12, 7-8. doi: $10.1038 /$ nmeth. 3213 
Yu, Z., Reichheld, S. E., Savchenko, A., Parkinson, J., and Davidson, A. R. (2010). A comprehensive analysis of structural and sequence conservation in the TetR family transcriptional regulators. J. Mol. Biol. 400, 847-864. doi: 10.1016/j.jmb. 2010.05.062

Zhu, J., Sun, D., Liu, W., Chen, Z., Li, J., and Wen, Y. (2016). AvaR2, a pseudo $\gamma$-butyrolactone receptor homologue from Streptomyces avermitilis, is a pleiotropic repressor of avermectin and avenolide biosynthesis and cell growth. Mol. Microbiol. 102, 562-578. doi: 10.1111/mmi.1 3479

Zou, Z., Du, D., Zhang, Y., Zhang, J., Niu, G., and Tan, H. (2014). A $\gamma$-butyrolactone-sensing activator/repressor, JadR3, controls a regulatory mininetwork for jadomycin biosynthesis. Mol. Microbiol. 94, 490-505. doi: 10.1111/ mmi. 12752
Conflict of Interest: YM was employed by the company Leibniz Institute DSMZ German Collection of Microorganisms and Cell Cultures GmbH.

The remaining authors declare that the research was conducted in the absence of any commercial or financial relationships that could be construed as a potential conflict of interest.

Copyright (c) 2020 Handel, Kulik and Mast. This is an open-access article distributed under the terms of the Creative Commons Attribution License (CC BY). The use, distribution or reproduction in other forums is permitted, provided the original author(s) and the copyright owner(s) are credited and that the original publication in this journal is cited, in accordance with accepted academic practice. No use, distribution or reproduction is permitted which does not comply with these terms. 